\title{
An audit of post-insertion fasting times following placement of a percutaneous endoscopic gastrostomy (PEG) tube
}

\author{
A. McHugh ${ }^{1}$, C. Browne ${ }^{1}$, S. Brady ${ }^{1}$, Y. Lydon ${ }^{1}$, D. Mc Cormack ${ }^{1}$ and J. Willow ${ }^{1}$ \\ ${ }^{1}$ Department of Clinical Nutrition, St. James's Hospital, Dublin 8, Ireland and ${ }^{2}$ University of Ulster, Coleraine, \\ Londonderry BT521SA
}

Placement of PEG tubes for nutrition support is an established practice. In recent years, recommendations have been published on when enteral feeding should commence following PEG tube placement (see below).

\begin{tabular}{lcc}
\hline Professional body & ASPEN $^{(1)}$ & NICE $^{(2)}$ \\
\hline Guideline/recommendation & A PEG tube may be utilised for feeding within several & In an uncomplicated patient there is no reason to delay \\
hours of placement: current iterature supports within & the start of feeding for more than 4 h after insertion \\
of a new PEG tube & $2 \mathrm{~h}$ in adults (Grade B) & \\
\hline
\end{tabular}

St James's Hospital is the largest academic teaching hospital in Ireland and a designated centre of excellence for cancer care. The objective of this audit was to review current practice in our institution and compare to published recommendations. Ethical approval was obtained prior to commencement of the audit.

Data were collected prospectively over an eight-month period. Primary PEG tube placements were carried out in endoscopy or theatre. Information collected included patient demographics, diagnosis and need for total or supplementary enteral tube feeding. Post-insertion instructions provided by the service placing the tube on when to commence feed were recorded and also details on when enteral feed actually commenced.

During the period audited, a total of 50 primary PEG tubes were placed. Sixty percent $(n$ 30) of the patients were male and the mean age was 74 years (range 32-92 years). Indications for PEG tube placement included cerebrovascular accident CVA $38 \%$ ( $n$ 19), malnutrition $22 \%(n 11)$, neurological $14 \%(n 7)$, upper-GI cancer $12 \%(n 6)$ and head and neck cancer $10 \%(n 5)$. The PEG tube was the sole source of nutrition support in $62 \%$ ( $n$ 31) of cases. Post-insertion fasting instructions were given by the medical team in $86 \%$ ( $n$ 43) of the cases (see below).

\begin{tabular}{lccccc}
\hline Fasting time instructed $(\mathrm{h})$ & 8 & 12 & 24 & Non-specific & 72 \\
\hline$\%$ (number) & $2 \%(n 1)$ & $46 \%(n 23)$ & $16 \%(n 8)$ & $2 \%(n 1)$ & $20 \%(n 10)$ \\
\hline
\end{tabular}

Delay in commencing enteral feed for patients where specific post-insertion instructions were provided occurred in $16 \%$ ( $n$ 7) of cases. Documented delays were up to $24 \mathrm{~h}$. Feeding was delayed appropriately in three of these cases due to medical reasons.

The findings from this audit show variation in instructions for fasting times post PEG tube placement across the institution. Enteral feeding was not commenced earlier than advised for any patient. In all cases fasting times were longer than international recommendations stated above. Further delays occurred in a number of cases. Delays in starting PEG feeding following insertion of a PEG tube may result in unnecessary prolongation of hospital stay and $\operatorname{costs}^{(2)}$. It is anticipated that collaboration between all stakeholders will enable development and implementation of an evidence-based protocol on fasting times post PEG placement in this hospital in the near future.

1. Bankhead R, Boullata J, Brantley S et al. (2009) Enteral nutrition practice recommendations. JPEN J Parenter Enteral Nutr 33, $122-167$.

2. National Collaborating Centre for Acute Care (2006) Nutrition Support in Adults: Oral Nutrition Support, Enteral Tube Feeding and Parenteral Nutrition. London: National Collaborating Centre for Acute Care, pp. 86-87. 